\title{
A counterexample to the Alon-Saks-Seymour conjecture and related problems
}

\author{
Hao Huang * $\quad$ Benny Sudakov ${ }^{\dagger}$
}

\begin{abstract}
Consider a graph obtained by taking edge disjoint union of $k$ complete bipartite graphs. Alon, Saks and Seymour conjectured that such graph has chromatic number at most $k+1$. This well known conjecture remained open for almost twenty years. In this paper, we construct a counterexample to this conjecture and discuss several related problems in combinatorial geometry and communication complexity.
\end{abstract}

\section{Introduction}

Tools from linear algebra have many striking applications in the study of combinatorial problems. One of the earliest such examples is the theorem of Graham and Pollak [7]. Motivated by a communication problem that arose in connection with data transmission, they proved that the edge set of a complete graph $K_{k}$ cannot be partitioned into disjoint union of less than $k-1$ complete bipartite graphs. Their original proof used Sylvester law of inertia. Over the years, this elegant result attracted a lot of attention and by now it has several different algebraic proofs, see [4, 15, 18, 20]. On the other hand no purely combinatorial proof of this statement is known.

A natural generalization of Graham-Pollak theorem is to ask whether the same estimate holds also for all graphs with chromatic number $k$. This problem was raised twenty years ago by Alon, Saks and Seymour who made the following conjecture (see, e.g., survey of J. Kahn, [9]).

Conjecture 1.1 If the edges of a graph $G$ can be partitioned into $k$ edge disjoint complete bipartite graphs, then the chromatic number of $G$ is at most $k+1$.

This question is also related to another long-standing open problem by Erdős, Faber and Lovász. They conjectured that the edge disjoint union of $k$ complete graphs of order $k$ is $k$-chromatic. Indeed, by replacing cliques in this problem by complete bipartite graphs we obtain the Alon-Saks-Seymour conjecture. The question of Erdős, Faber and Lovász is still open. On the other hand, Kahn [8] proved the asymptotic version of their conjecture, showing that the chromatic number of edge disjoint union of $k$ complete graphs of order $k$ has chromatic number at most $(1+o(1)) k$.

Let $\mathbf{b p}(G)$ be the minimum number of bicliques (i.e., complete bipartite graphs) needed to partition the edges of graph $G$ and $\chi(G)$ be the chromatic number of $G$. The Alon-Saks-Seymour

\footnotetext{
*Department of Mathematics, UCLA, Los Angeles, CA, 90095. Email: huanghao@math.ucla.edu.

${ }^{\dagger}$ Department of Mathematics, UCLA, Los Angeles, CA 90095. Email: bsudakov@math.ucla.edu. Research supported in part by NSF CAREER award DMS-0812005 and by a USA-Israeli BSF grant.
} 
Conjecture can be restated as $\mathbf{b p}(G) \geq \chi(G)-1$. Until recently, there was not much known about this conjecture. Using folklore result that the chromatic number of the union of graphs is at most the product of their chromatic numbers, one can easily get a lower bound $\mathbf{b p}(G) \geq \log _{2} \chi(G)$. In [13, Mubayi and Vishwanathan improved the lower bound to $2^{\sqrt{2 \log _{2} \chi(G)}}$. This estimate can be also deduced from the well known result of Yannakakis [21] in communication complexity. This connection to communication complexity was discovered by Alon and Haviv 2] (see Section 4 for details). Gao, McKay, Naserasr and Stevens [6] introduced a reformulation of the Alon-Saks-Seymour conjecture and verified it for graphs with chromatic number $k \leq 9$. The main aim of this paper is to obtain a superlinear gap between chromatic number and biclique partition number, which disproves the Alon-Saks-Seymour conjecture.

Theorem 1.2 There exists an infinite collection of graphs $G$ such that $\chi(G) \geq c(\mathbf{b p}(G))^{6 / 5}$, for some fixed constant $c>0$.

The study of (two-party) communication complexity, introduced by Yao [22], is an important topic in theoretical computer science which has many applications. In the basic model we have two players Alice and Bob who are trying to evaluate a boolean function $f: X \times Y \rightarrow\{0,1\}$. Alice only knows $x$, Bob only knows $y$ and thus they want to communicate with each other according to some fixed protocol in order to compute $f(x, y)$. The goal is to minimize the amount of communication during the protocol. The deterministic communication complexity $D(f)$ is the number of bits that needs to be exchanged for the worst inputs $x, y$ by the best protocol for $f$. Let $M$ be a matrix of $f$, i.e., $M_{x, y}=f(x, y)$ and let $r k(M)$ be the rank of $M$. It's known that $D(f) \geq \log _{2} r k(M)$. Lovász and Saks [12] conjectured that this bound is not very far from being tight. More precisely, their log-rank conjecture says that $D(f) \leq\left(\log _{2} r k(M)^{O(1)}\right.$. This problem is directly related to the rank-coloring conjecture of Van Nuffelen [19] and Fajtlowicz [5] in graph theory. This conjecture, which was disproved by Alon and Seymour [3], asked whether the chromatic number of a graph $G$ is bounded by the rank of its adjacency matrix $A_{G}$. It is known that separation result between $D(f)$ and $\log _{2} r k(M)$ give corresponding separation between $\chi(G)$ and $r k\left(A_{G}\right)$. Several authors gave such separation results, e.g., [17, 16]. So far, the largest gap was obtained by Nisan and Wigderson [14] who constructed an infinite family of matrices such that $D(f)>\left(\log _{2} r k(M)\right)^{\log _{2} 3}$.

Similar to the rank-coloring problem, the Alon-Saks-Seymour conjecture is also closely related to a well known open problem in communication complexity. This communication problem, which is called clique versus independent set ( $C L-I S$ for brevity), was introduced by Yannakakis [21] in 1988. In this problem, there is a publicly known graph $G$, Alice gets a clique $C$ of $G$ and Bob gets an independent set $I$ of $G$. Their goal is to output $|C \cap I|$, which is clearly either 0 or 1 . We will discuss connection between this problem and the Alon-Saks-Seymour conjecture and show that our counterexample yields a first nontrivial lower bound on the non-deterministic communication complexity of $C L-I S$ problem.

The rest of this short paper is organized as follows. In the next section we describe a counterexample to the Alon-Saks-Seymour Conjecture. In Section 3, we consider minimal coverings of a graph by bicliques in which every edge of the graph is covered at least once and at most $t$ times, for some parameter $t$. This more general notion is closely related to the question in combinatorial geometry about a neighborly family of boxes. We show that a natural variant of the Alon-Saks-Seymour 
conjecture for this more general parameter fails as well. In Section 4, we discuss connections with communication complexity and use our counterexample to obtain a new lower bound on nondeterministic communication complexity of clique vs. independent set problem. The final section contains some concluding remarks and open problems.

Notation. The $n$-dimensional cube $Q_{n}$ is $\{0,1\}^{n}$ and two vertices $x, y$ of $Q_{n}$ are adjacent $x \sim y$ if and only if they differ in exactly one coordinate. A $k$-dimensional subcube of $Q_{n}$ is a subset of $\{0,1\}^{n}$ which can be written as $\left\{x=\left(x_{1}, \cdots, x_{n}\right) \in Q_{n}: x_{i}=a_{i}, \forall i \in T\right\}$, where $T$ is a set of $n-k$ coordinates (called fixed coordinates), each $a_{i}$ is a fixed element in $\{0,1\}$. In addition, we write $1^{n}$ and $0^{n}$ to represent the all-one and all-zero vector in $Q_{n}$ and use $Q_{n}^{-}$to indicate the set $Q_{n} \backslash\left\{1^{n}, 0^{n}\right\}$. Given two subset $X \subset Q_{k}$ and $Y \subset Q_{\ell}$ we denote by $X \times Y$ a subset of cube $Q_{k+\ell}$ which consists of all binary vectors $(x, y)$ with $x \in X$ and $y \in Y$.

For graph $G=(V, E)$ with vertex set $V$ and edge set $E$, we denote by $\chi(G), \alpha(G), \mathbf{b p}(G)$ the chromatic number, independence number and biclique partition number respectively. The collection of all independent sets in $G$ is denoted by $\mathcal{I}(G)$. Similarly $\mathcal{C}(G)$ stands for the set of all cliques in $G$. The $O R$ product of two graphs $G$ and $H$ is defined as a graph with vertex set equal to the Cartesian product $V(G) \times V(H)$, two vertices $(g, h) \sim\left(g^{\prime}, h^{\prime}\right)$ iff $g \sim g^{\prime}$ in $G$ or $h \sim h^{\prime}$ in $H$. The $m$-blowup of a graph $G$ is obtained by replacing every vertex $v$ of $G$ with an independent set $I_{v}$ of size $m$ and by replacing every edge $(u, v)$ of $G$ with a complete bipartite graph, whose parts are the independent sets $I_{u}$ and $I_{v}$. We also use the notation $\mathcal{B}(U, W)$ to indicate a biclique with two parts $U$ and $W$.

To state asymptotic results, we utilize the following standard notations. For two functions $f(n)$ and $g(n)$, write $f(n)=\Omega(g(n))$ if there exists a positive constant $c$ such that $\liminf _{n \rightarrow \infty} f(n) / g(n) \geq$ $c, f(n)=o(g(n))$ if $\limsup _{n \rightarrow \infty} f(n) / g(n)=0$. Also, $f(n)=O(g(n))$ if there exists a positive constant $C>0$ such that $\lim _{\sup _{n \rightarrow \infty}} f(n) / g(n) \leq C$.

\section{Main Result}

In this section we describe a counterexample to the Alon-Saks-Seymour conjecture. Our construction is inspired by and is somewhat similar to Razborov's counterexample to the rank-coloring conjecture [17. Consider the following graph $G=(V, E)$. Its vertex set is $V(G)=[n]^{7}=\left\{\left(x_{1}, \cdots, x_{7}\right): x_{i} \in\right.$ $[n]\}$. For any two vertices $x=\left(x_{1}, \cdots, x_{7}\right), y=\left(y_{1}, \cdots, y_{7}\right)$ in $V(G)$, let $\rho$ be the comparing function which records all coordinates in which they differ. More precisely, $\rho(x, y)=\left(\rho_{1}(x, y), \cdots, \rho_{7}(x, y)\right) \in$ $Q_{7}$, such that

$$
\rho_{i}(x, y)= \begin{cases}1 & \text { if } x_{i} \neq y_{i} \\ 0 & \text { if } x_{i}=y_{i}\end{cases}
$$

Two vertices $x$ and $y$ are adjacent in $G$ if and only if $\rho(x, y) \in S$, where $S$ is the following subset of the cube $Q_{7}$

$$
S=Q_{7} \backslash\left[\left(1^{4} \times Q_{3}^{-}\right) \cup\left\{0^{4} \times 0^{3}\right\} \cup\left\{0^{4} \times 1^{3}\right\}\right] .
$$

In the rest of this section we show that this graph $G$ satisfies the assertion of Theorem 1.2 .

Proposition 2.1 The independence number of $G$ satisfies $\alpha(G)=O(n)$. 
Proof. Let $I$ be an independent set in $G$. For any set of indices $T=\left\{i_{1}, \ldots, i_{t}\right\} \subset\{1,2, \cdots, 7\}$, let $p_{T}$ be the natural projection of $[n]^{7}$ to $[n]^{T}$. For every vector $x \in[n]^{7}$ it outputs the restriction of $x$ to the coordinates in $T$, i.e., $p_{T}(x)=\left(x_{i_{1}}, \ldots, x_{i_{t}}\right)$. For convenience, we will for example write $p_{1234}$ instead of $p_{\{1,2,3,4\}}$. It is easy to check from the definition of $S$, that any two vertices $x, y \in G$ which agree on one of the first 4 coordinates and satisfy $p_{1234}(x) \neq p_{1234}(y)$ are adjacent in $G$. Hence, any two vectors in $p_{1234}(I)$ differ in all their coordinates and therefore $\left|p_{1234}(I)\right| \leq n$. If in addition, we also have for every element $x \in p_{1234}(I),\left|p_{1234}^{-1}(x) \cap I\right| \leq 3$, then $|I| \leq 3\left|p_{1234}(I)\right|=O(n)$ and the proof is complete.

Otherwise, we may assume the existence of $\widetilde{x} \in[n]^{4}$ and different vertices $\widetilde{x}_{1}, \widetilde{x}_{2}, \widetilde{x}_{3}, \widetilde{x}_{4} \in I$ such that $p_{1234}\left(\widetilde{x}_{i}\right)=\widetilde{x}$. By the definition of $S$, it is easy to see that $p_{567}\left(\widetilde{x}_{i}\right)$ differ in every coordinate. Since $1^{7} \in S$, we have that any two vertices of $G$ which differ in all 7 coordinates are adjacent. This implies that if there is a vertex $z \in I$ with $p_{1234}(z)$ different from $\widetilde{x}$, then $p_{567}(z)$ and $p_{567}\left(\widetilde{x}_{i}\right)$ are equal in at least one coordinate. Since the number of coordinates of $p_{567}(I)$ is only 3 and there are 4 vertices $\widetilde{x}_{1}, \widetilde{x}_{2}, \widetilde{x}_{3}, \widetilde{x}_{4}$, we have that two of these vertices agree with $p_{567}(I)$ (and hence with each other) in the same coordinate. This contradicts the fact that $p_{567}\left(\widetilde{x}_{i}\right)$ differ in all coordinates, and implies that there is only one element in $p_{1234}(I)$. Again, by the definition of $S$, the vertices in $I$ are different in each of the last three coordinates. As a result $|I|=\left|p_{567}(I)\right| \leq n$.

Corollary 2.2 The chromatic number of $G$ is at most $\Omega\left(n^{6}\right)$.

Proof. Apply Proposition 2.1 together with the well-known fact that $\chi(G) \geq \frac{|V(G)|}{\alpha(G)}$.

Proposition 2.3 The biclique partition number satisfies $\mathbf{b p}(G)=O\left(n^{5}\right)$.

Before going into the details of the proof of this statement, first we need the following two lemmas.

Lemma 2.4 $S$ can be partitioned into disjoint union $S=\cup_{i=1}^{30} S_{i}$, where $S_{i}$ are 2 -dimensional subcubes in $Q_{7}$.

Proof. We need the following simple observations.

(a) $Q_{3}^{-}$is a disjoint union of 1 -dimensional subcubes.

(b) $Q_{3}$ can be decomposed into disjoint union of 2-dimensional subcubes.

(c) For every $R_{1} \subset Q_{4}$, the set $R_{1} \times Q_{3}$ can be decomposed into disjoint union of 2-dimensional subcubes.

(d) For any $x_{1} \sim x_{2}$ in $Q_{4}, y_{1} \sim y_{2}$ in $Q_{3},\left(x_{1}, y_{1}\right),\left(x_{1}, y_{2}\right),\left(x_{2}, y_{1}\right),\left(x_{2}, y_{2}\right)$ is a 2 -dimensional subcube in $Q_{7}$.

(e) For any $x_{1} \sim x_{2}$ in $Q_{4},\left(x_{1} \times Q_{3}^{-}\right) \cup\left(x_{2} \times Q_{3}^{-}\right)$can be decomposed into disjoint union of 2-dimensional subcubes.

To verify $(a)$ note that, $Q_{3}^{-}=\{(0,0,1),(0,1,1)\} \cup\{(0,1,0),(1,1,0)\} \cup\{(1,0,0),(1,0,1)\}$. Claims $(b)$ and $(d)$ are obvious by the definition of cubes. Claims $(c)$ is an immediate corollary of $(b)$, and claim $(e)$ follows easily from $(a)$ and $(d)$. 
Next we can partition the set $S=Q_{7} \backslash\left[\left(1^{4} \times Q_{3}^{-}\right) \cup\left\{0^{4} \times 0^{3}\right\} \cup\left\{0^{4} \times 1^{3}\right\}\right]$ into the following 3 disjoint subsets $S^{\prime}, S^{\prime \prime}, S^{\prime \prime \prime}$ and show that each of them is itself a disjoint union of 2-dimensional subcubes.

$$
S^{\prime}=\left\{\begin{array}{l}
(0,0,0,0) \times Q_{3}^{-} \cup(0,0,0,1) \times Q_{3}^{-} \\
(0,0,1,1) \times Q_{3}^{-} \cup(1,0,1,1) \times Q_{3}^{-} \\
(0,1,0,1) \times Q_{3}^{-} \cup(0,1,1,1) \times Q_{3}^{-} \\
(1,1,0,1) \times Q_{3}^{-} \cup(1,0,0,1) \times Q_{3}^{-}
\end{array}\right.
$$

This set can be partitioned into disjoint union of 2-dimensional subcubes, using claim $(e)$.

$$
S^{\prime \prime}=\left\{\begin{array}{l}
(1,1,1,1) \times 0^{3} \cup(1,1,0,1) \times 0^{3} \cup(1,0,1,1) \times 0^{3} \cup(1,0,0,1) \times 0^{3} \\
(1,1,1,1) \times 1^{3} \cup(1,1,0,1) \times 1^{3} \cup(1,0,1,1) \times 1^{3} \cup(1,0,0,1) \times 1^{3} \\
(0,1,1,1) \times 0^{3} \cup(0,1,0,1) \times 0^{3} \cup(0,0,1,1) \times 0^{3} \cup(0,0,0,1) \times 0^{3} \\
(0,1,1,1) \times 1^{3} \cup(0,1,0,1) \times 1^{3} \cup(0,0,1,1) \times 1^{3} \cup(0,0,0,1) \times 1^{3}
\end{array}\right.
$$

Note that, every line in the definition of $S^{\prime \prime}$ describes a 2-dimensional subcube. This shows that $S^{\prime \prime}$ is a disjoint union of four 2-dimensional subcubes.

$$
S^{\prime \prime \prime}=\left\{\begin{array}{l}
(0,0,1,0) \times Q_{3} \cup(0,1,0,0) \times Q_{3} \cup(1,0,0,0) \times Q_{3} \cup(0,1,1,0) \times Q_{3} \\
(1,0,1,0) \times Q_{3} \cup(1,1,0,0) \times Q_{3} \cup(1,1,1,0) \times Q_{3}
\end{array}\right.
$$

To decompose this set into disjoint union of 2-dimensional subcubes, one can use claim $(c)$.

Finally, it is easy to verify that indeed $S=S^{\prime} \cup S^{\prime \prime} \cup S^{\prime \prime \prime}$ and hence $S$ can be partitioned into 2-dimensional subcubes.

Using the decomposition $S=\cup_{i=1}^{30} S_{i}$ from Lemma 2.4, we can define the following subgraphs $G_{i} \subset G$. The vertex set $V\left(G_{i}\right)=V(G)$ and two vertices $x, y \in G_{i}$ are adjacent if and only if $\rho(x, y) \in S_{i}$. From this definition, it is easy to see that $G$ is the edge disjoint union of subgraphs $G_{i}$. Next we will show that every $G_{i}$ has a small biclique partition number.

Lemma $2.5 \mathrm{bp}\left(G_{i}\right) \leq n^{5}$.

Proof. Recall that the set $S_{i}$, which is used to define edges of $G_{i}$, is a 2-dimensional subcube of $Q_{7}$. Therefore there exists a set $T=\left\{t_{1}, \ldots, t_{5}\right\} \subset\{1, \cdots, 7\}$ of fixed coordinates and $a_{1}, \ldots, a_{5} \in\{0,1\}$, such that $S_{i}=\left\{x=\left(x_{1}, \cdots, x_{7}\right): x_{t_{j}}=a_{j}, \forall 1 \leq j \leq 5\right\}$. Now we define graph $\widetilde{G}_{i}$. Its vertex set $V\left(\widetilde{G}_{i}\right)=[n]^{5}$ and two vertices $\widetilde{x}$ and $\widetilde{y}$ are adjacent in $\widetilde{G}_{i}$ if an only if $\rho(\widetilde{x}, \widetilde{y})=\left(a_{1}, \ldots, a_{5}\right)$. It is rather straightforward to see that $G_{i}$ is a $n^{2}$-blowup of $\widetilde{G}_{i}$.

To complete the proof of this lemma we need two basic facts about biclique partition number. The first one says that for any graph $H, \mathbf{b} \mathbf{p}(H) \leq|V(H)|-1$. Indeed, removing stars rooted at every vertex, one by one, we can partition every graph on $h$ vertices into $h-1$ bicliques. The second one, claims that if $H$ is a blowup of $\widetilde{H}$, then $\mathbf{b p}(H) \leq \mathbf{b} \mathbf{p}(\widetilde{H})$. To prove this, note that the blowup 
of biclique is a biclique itself. Therefore blowup of all the bicliques in a partition of $\widetilde{H}$ becomes a biclique partition of $H$.

These two statements, together with the fact (mentioned above) that $G_{i}$ is the blowup of $\widetilde{G}_{i}$, imply that $\mathbf{b p}\left(G_{i}\right) \leq \mathbf{b p}\left(\widetilde{G}_{i}\right) \leq\left|V\left(\widetilde{G}_{i}\right)\right|-1 \leq n^{5}$.

Proof of Proposition 2.3. Using that $G$ is the edge disjoint union of $G_{i}$ together with Lemma 2.5. we conclude that, $\mathbf{b p}(G)=\mathbf{b} \mathbf{p}\left(\cup_{i=1}^{30} G_{i}\right) \leq \sum_{i=1}^{30} \mathbf{b p}\left(G_{i}\right)=O\left(n^{5}\right)$.

Propositions 2.2 and 2.3 show that graph $G$, which we constructed, indeed satisfies the assertion of Theorem 1.2 and disproves the Alon-Saks-Seymour Conjecture.

\section{Neighborly family of boxes and $t$-biclique covering number}

The Alon-Saks-Seymour conjecture deals with the minimum number of bicliques needed to cover all the edges of a given graph $G$ exactly once. It is also very natural to consider a more general problem in which we are allowed to cover the edges of graph at most $t$ times. A $t$-biclique covering of a graph $G$ is a collection of bicliques that cover every edge of $G$ at least once and at most $t$ times. The minimum size of such covering is called the $t$-biclique covering number and is denoted by $\mathbf{b p}_{t}(G)$. In particular, $\mathbf{b p}_{1}(G)$ is the usual biclique partition number $\mathbf{b p}(G)$.

In addition to being an interesting parameter to study in its own right, the $t$-biclique covering number is also closely related to the question in combinatorial geometry about neighborly family of boxes. A finite family $\mathcal{C}$ of $d$-dimensional convex polytopes is called $t$-neighborly if $d-t \leq \operatorname{dim}(C \cap$ $\left.C^{\prime}\right) \leq d-1$ for every two distinct members $C$ and $C^{\prime}$ of $\mathcal{C}$. One particularly interesting case is when $\mathcal{C}$ consists of $d$-dimensional boxes with edges parallel to the coordinate axes. This type of box is called standard box. Using Graham-Pollak theorem, Zaks [23] proved that the maximum possible cardinality of a 1-neighborly family of standard boxes in $\mathbb{R}^{d}$ is precisely $d+1$. His result was generalized by Alon [1], who proved that $\mathbb{R}^{d}$ has a $t$-neighborly family of $k$ standard boxes if and only if the complete graph $K_{k}$ can has $t$-biclique covering of size $d$. This shows that the problem of determining the maximum possible cardinality of $t$-neighborly families of standard boxes and the problem of computing the $t$-biclique covering number of complete graphs are equivalent.

In his paper [1], Alon gave asymptotic estimates for $\mathbf{b} \mathbf{p}_{t}\left(K_{k}\right)$ showing that

$$
(1+o(1))\left(t ! / 2^{t}\right)^{1 / t} k^{1 / t} \leq \mathbf{b p}_{t}\left(K_{k}\right) \leq(1+o(1)) t k^{1 / t} .
$$

There is still gap between these two bounds and the problem of determining the right constant before $k^{1 / t}$ is wide open even for the case when $t=2$. Using a different proof, we obtain here a slightly better lower bound of order roughly $\left(t ! / 2^{t-1}\right)^{1 / t} k^{1 / t}$. For $t=2$ it improves the above estimate by a factor of $\sqrt{2}$.

Proposition 3.1 If there exists a t-biclique covering of $K_{k}$ of size $d$, then $k \leq 1+\sum_{s=1}^{t} 2^{s-1}\left(\begin{array}{l}d \\ s\end{array}\right)$.

Proof. Suppose that the edges of $K_{k}$ are covered by the bicliques $\left\{\mathcal{B}\left(U_{j}, W_{j}\right)\right\}_{j=1}^{d}$, such that every edge is covered at least once and at most $t$-times. For every nonempty subset of indices $S \subset[d]$ of size 
$|S| \leq t$ let $H_{S}=\cap_{j \in S} \mathcal{B}\left(U_{j}, W_{j}\right)$ and let $A_{S}$ be the adjacency matrix of $H_{S}$. Let $J$ be $k \times k$ matrix of ones and let $I$ be the $k \times k$ identity matrix. Then $J-I$ is the adjacency matrix of $K_{k}$ and it is easy to see, using the inclusion-exclusion principle, that

$$
J-I=\sum_{S \subset[d], 0<|S| \leq t}(-1)^{|S|} A_{S} .
$$

Also note that for $|S|=s$, the graph $H_{S}$ is the disjoint union of at most $2^{s-1}$ smaller bicliques. Indeed, for every binary vector $z=\left(z_{1}, \ldots, z_{s-1}\right)$ consider a complete bipartite graph with parts

$$
X_{z}=\cap_{j, z_{j}=0} U_{j} \cap_{j, z_{j}=1} W_{j} \cap U_{s} \text { and } Y_{z}=\cap_{j, z_{j}=0} W_{j} \cap_{j, z_{j}=1} U_{j} \cap W_{s} .
$$

It is not difficult to check that these bicliques are disjoint and their union is $H_{S}$. Therefore, for every $S \subset[d], 0<|S|=s \leq t$ we can write $A_{S}=\sum_{i} B_{i, S}$ where $B_{i, S}$ is an adjacency matrix of a biclique and $1 \leq i \leq 2^{s-1}$. Thus we obtain that $J-I$ can be written as a linear combination of at most $m=\sum_{s=1}^{t} 2^{s-1}\left(\begin{array}{l}d \\ s\end{array}\right)$ adjacency matrices of complete bipartite graphs.

Now to complete the proof we use the elegant trick of Peck [15] (we can use here other known proofs of Graham-Pollak theorem as well). For bipartite graph with adjacency matrix $B_{i, S}$ let $B_{i, S}^{\prime}$ be $k \times k$ matrix which contains only ones in positions whose row index lies in the first part of the bipartition and whose column index lies in the second part of the bipartition, the rest of the entries of $B_{i, S}^{\prime}$ are zeros. Since the corresponding bipartite graph is complete, $B_{i, S}^{\prime}$ has rank one. Furthermore, the matrix $B_{i, S}-2 B_{i, S}^{\prime}$ is antisymmetric. As a result we can write $J-I$ as a linear combination of at most $m$ rank one matrices plus some antisymmetric matrix $T$. Since an antisymmetric real matrix has only imaginary eigenvalues, $I+T$ must have a full rank $k$. But its rank can not exceed the rank of the linear combination of at most $m$ rank one matrices plus $J$. As $J$ has rank one as well, this implies that $k \leq m+1=1+\sum_{s=1}^{t} 2^{s-1}\left(\begin{array}{l}d \\ s\end{array}\right)$ and completes the proof.

As we already mentioned in the introduction, the motivation for the Alon-Saks-Seymour conjecture comes from Graham-Pollak theorem which says that $\mathbf{b p}\left(K_{k}\right) \geq k-1$. Similarly, based on the lower bound of Alon that $\mathbf{b p}_{t}\left(K_{k}\right) \geq \Omega\left(k^{1 / t}\right)$, one can consider the following very natural generalization of this conjecture.

Question 3.2 Is it true that for every fixed integer $t>0$, there exist a constant $c=c(t)$ such that $\mathbf{b p}_{t}(G) \geq c(\chi(G))^{1 / t}$ for all graphs $G$ ?

Recall that in Section 2 we constructed a graph $G$ with $|V(G)|=n^{7}$ vertices such that $\alpha(G)=O(n)$ and $\mathbf{b p}(G)=O\left(n^{5}\right)$. Consider the $O R$ product (defined in the introduction) of $t$ copies of $G$. We show that the graph $G^{t}$ gives a negative answer to the above question for all positive integers $t$. This follows from the following sequence of claims.

Claim 3.3 $\alpha\left(G^{t}\right) \leq \alpha(G)^{t}=O\left(n^{t}\right)$.

Proof. We only need to prove $\alpha(G \times H) \leq \alpha(G) \alpha(H)$ for any two graphs $G$ and $H$, since then the claim follows by induction on $t$. To prove this statement, consider the maximum independent set $I \in G \times H$. Let $I^{\prime}=\{v \in G \mid(v, u) \in I$ for some $u \in H\}$ be the projection of $I$ on $V(G)$. By 
the definition of $O R$ product, this is an independent set in $G$ and therefore has size at most $\alpha(G)$. Similarly, if $I^{\prime \prime}$ is the projection of $I$ on $V(H)$ then $\left|I^{\prime \prime}\right| \leq \alpha(H)$. To complete the proof note that $I$ is a subset of $I^{\prime} \times I^{\prime \prime}$ and therefore its size cannot exceed $\alpha(G) \alpha(H)$.

Corollary $3.4 \chi\left(G^{t}\right)=\Omega\left(n^{6 t}\right)$.

Proof. By Claim 3.3, $\chi\left(G^{t}\right) \geq \frac{\left|V\left(G^{t}\right)\right|}{\alpha\left(G^{t}\right)} \geq \frac{n^{7 t}}{\alpha(G)^{t}}=\Omega\left(n^{6 t}\right)$.

Claim $3.5 \mathbf{b p}_{t}\left(G^{t}\right) \leq t \mathbf{b p}(G)$.

Proof. Consider graphs $H_{i}, 1 \leq i \leq t$ with vertex set $V\left(H_{i}\right)=V\left(G^{t}\right)$ such that two vertices $\left(h_{1}, \cdots, h_{t}\right)$ and $\left(h_{1}^{\prime}, \cdots, h_{t}^{\prime}\right)$ are adjacent in $H_{i}$ if and only if $h_{i} \sim h_{i}^{\prime}$ in $G$. Note that $H_{i}$ is an $n^{t-1}$-blowup of $G$ and therefore $\mathbf{b} \mathbf{p}\left(H_{i}\right)=\mathbf{b} \mathbf{p}(G)$. Also it is easy to see that every edge in $G^{t}$ is covered by some $H_{i}$. Since the number of graphs $H_{i}$ is $t$, every edge of $G^{t}$ is covered at most $t$ times. Then the union of minimum biclique partitions of all $H_{i}$ gives a $t$-biclique covering of $G$. Hence $\mathbf{b p}_{t}\left(G^{t}\right) \leq \sum_{i=1}^{t} \mathbf{b p}\left(H_{i}\right) \leq t \mathbf{b} \mathbf{p}(G)$.

Claim $3.6 \mathbf{b p}_{t}\left(G^{t}\right) \leq c\left(\chi\left(G^{t}\right)\right)^{\frac{5}{6 t}}$ for some constant $c=c(t)$.

Proof. By Claims 3.4 and 3.5, $\mathbf{b p}_{t}\left(G^{t}\right) \leq t \mathbf{b p}(G)=O\left(t n^{5}\right) \leq c(t)\left(\chi\left(G^{t}\right)\right)^{\frac{5}{6 t}}$.

This shows that the answer to the Question 3.2 is negative for all natural $t$.

\section{Clique vs. independent set communication problem}

In the introduction, we already defined the two-party communication model and discussed the concept of deterministic communication complexity. Here we need a few additional notions and definitions (see e.g., [1] for more details). The non-deterministic communication complexity $N^{1}(f)$ of a function $f$ is the smallest number of bits needed by an all powerful prover to convince Alice and Bob that $f(x, y)=1$. It is known that $N^{1}(f)=\left\lceil\log _{2} C^{1}(f)\right\rceil$, where $C^{1}(f)$ is the minimum number of monochromatic combinatorial rectangles needed to cover the 1-inputs of communication matrix $M$ of $f$ (recall that $M_{x, y}=f(x, y)$ ). With slight abuse of notation we will later write $C^{1}(M)$ instead of $C^{1}(f)$. The numbers $N^{0}(f), C^{0}(f), C^{0}(M)$ are defined similarly, and the relation $N^{0}(f)=\left\lceil\log _{2} C^{0}(f)\right\rceil$ holds as well.

In this section we consider the communication complexity of the clique versus independent set problem $(C L-I S)$. In this problem, there is a publicly known graph $\Gamma$, Alice gets a clique $C$ of $\Gamma$ and Bob gets an independent set $I$ of $\Gamma$. Their goal is to output $|C \cap I|$, which is clearly either 0 or 1 . This problem was first introduced by Yannakakis [21, who also proposed the following algorithm to solve it. Given a graph $\Gamma$ on $m$ vertices, Alice sends to Bob a name of the vertex $v$ in $C$ whose degree in $\Gamma$ is at most $m / 2$. Note that in this case we can reduce the size of the graph by a factor of two by looking only on the subgraph $\Gamma^{\prime}$ induced by the neighbors of $v$. Bob in his turn send Alice a name of 
the vertex $u$ in his independent set $I \cap \Gamma^{\prime}$ which has degree at least $\left|V\left(\Gamma^{\prime}\right)\right| / 2$. Also in this case we can reduce the size of the remaining problem by a factor of two. Finally if both Alice and Bob can not send anything it is easy to see that $C \cap I=\emptyset$. By repeating this procedure at $\operatorname{most} \log _{2} m$ rounds, one can show that the deterministic communication complexity satisfies $D\left(C L-I S_{\Gamma}\right) \leq O\left(\log _{2}^{2} m\right)$. However, so far the best lower bound for this problem (see [10]) is only asymptotically $2 \log _{2} m$.

For non-deterministic communication complexity of clique vs. independent set problem, it's easy to see that $N^{1}\left(C L-I S_{\Gamma}\right)$ is always $\log m$. Indeed, for every vertex $v \in \Gamma$ consider the rectangle $R_{v}$ formed by all cliques vs. all independent sets containing $v$. By definition, these $m$ rectangles cover all 1-inputs of the communication matrix $M$ of $C L-I S_{\Gamma}$. On the other hand, determining the correct order of magnitude of $N^{0}\left(C L-I S_{\Gamma}\right)$ is wide open except for the trivial lower bound $\log _{2} m$. This lower bound follows from the simple fact that taking all single vertices as cliques vs. the same vertices as independent sets shows that the $m \times m$ identity matrix is a submatrix of $M$. Next we discuss the connection between the Alon-Saks-Seymour conjecture and $C L-I S$ problem which was discovered by Alon and Haviv [2]. This connection together with our counterexample gives a first nontrivial lower bound for nondeterministic communication complexity of clique vs. independent set problem. It implies that there exists a graph $\Gamma$ such that $N^{0}\left(C L-I S_{\Gamma}\right) \geq 6 / 5 \log _{2} m-O(1)$.

Suppose we have a graph $G=(V, E), V(G)=[n], \mathbf{b p}(G)=m$ and a partition of $E(G)$ as disjoint union of bicliques $\left\{\mathcal{B}\left(U_{i}, W_{i}\right)\right\}_{i=1}^{m}$. Define the characteristic vector $v_{i}$ of each biclique to be $v_{i}=\left(v_{i 1}, \cdots, v_{i n}\right) \in\{0,1, *\}^{n}$, so that

$$
v_{i j}= \begin{cases}0 & \text { if } j \in U_{i} \\ 1 & \text { if } j \in W_{i} \\ * & \text { otherwise }\end{cases}
$$

Using the notations above, we create a new graph $\Gamma$ on vertex set $[m]$. Two vertices $i$ and $i^{\prime}$ are adjacent in $\Gamma$ if there exists $j \in[n]$ such that $v_{i j}=v_{i^{\prime} j}=1$. Two vertices $i$ and $i^{\prime}$ are nonadjacent if there exists $j^{\prime} \in[n]$ such that $v_{i j^{\prime}}=v_{i^{\prime} j^{\prime}}=0$. In any other case, arbitrarily assign an edge or non-edge between $i$ and $i^{\prime}$. If there are two indices $j, j^{\prime}$ such that $v_{i j}=v_{i^{\prime} j}=1$ and $v_{i j^{\prime}}=v_{i^{\prime} j^{\prime}}=0$, then $j \in W_{i} \cap W_{i^{\prime}}$ and $j^{\prime} \in U_{i} \cap U_{i^{\prime}}$. Therefore the edge $\left(j^{\prime}, j\right)$ is covered by two bicliques, which is impossible since $\cup_{i=1}^{m} \mathcal{B}\left(U_{i}, W_{i}\right)$ is an edge partition of $G$. This shows that $\Gamma$ is well defined.

Now consider the $C L-I S$ problem on $\Gamma$. Define $C_{j}=\left\{q \in[m]: v_{q j}=1\right\}$ and $I_{j}=\{q \in[m]$ : $\left.v_{q j}=0\right\}$. By definition of $\Gamma$, it is easy to see that $\left\{C_{j}\right\}$ are cliques and $\left\{I_{j}\right\}$ are independent sets in this graph. Denote the matrix of $C L-I S_{\Gamma}$ by $M$. Let $M^{\prime}$ be a submatrix of $M$ corresponding to the rows determined by $\left\{C_{j}\right\}_{j=1}^{n}$ and columns determined by $\left\{I_{j}\right\}_{j=1}^{n}$. Obviously $N^{0}(M) \geq N^{0}\left(M^{\prime}\right)=$ $\log _{2} C^{0}\left(M^{\prime}\right)$. Assume that we have a covering of 0 -entries of $M^{\prime}$ by monochromatic rectangles, and let $R_{1}, \cdots, R_{t}$ be the rectangles which cover the diagonal entries of $M^{\prime}$. Note that if $(p, q)$ is covered by $R_{i}$, then $M_{p q}^{\prime}=M_{q p}^{\prime}=0$ and thus $C_{p} \cap I_{q}$ and $C_{q} \cap I_{p}$ are both empty. This implies that $(p, q)$ is not an edge in graph $G$, since otherwise there must exist an index $i$ such that $v_{i p}=0, v_{i q}=1$ or $v_{i p}=1, v_{i q}=0$. Then either $i \in I_{p} \cap C_{q}$ or $i \in C_{p} \cap I_{q}$, which gives a contradiction. In particular, the family of rectangles $\left\{R_{i}\right\}_{i=1}^{t}$ corresponds to a covering of graph $G$ by independent sets and therefore $\chi(G) \leq t$. Thus we have that

$$
N^{0}(M) \geq N^{0}\left(M^{\prime}\right)=\log _{2} C^{0}\left(M^{\prime}\right) \geq \log _{2} t \geq \log _{2} \chi(G) .
$$


This estimate together with the existence of a graph $G$ (from Section 2) which has $\mathbf{b p}(G)=$ $O\left(\chi(G)^{5 / 6}\right)$, proves the following theorem.

Theorem 4.1 There exists an infinite collection of graphs $\Gamma$, such that

$$
N^{0}\left(C L-I S_{\Gamma}\right) \geq \frac{6}{5} \log _{2}|V(\Gamma)|-O(1) .
$$

In addition, the combination of the inequality $N^{0}\left(C L-I S_{\Gamma}\right) \geq \log _{2} \chi(G)$ we just proved, and the result of Yannakakis that $D\left(C L-I S_{\Gamma}\right) \leq O\left(\log _{2}^{2} m\right)$, immediately gives a different derivation of the following result of Mubayi and Vishwanathan. It shows that if $\mathbf{b p}(G)=m$, then

$$
\chi(G) \leq 2^{N^{0}\left(C L-I S_{\Gamma}\right)} \leq 2^{D\left(C L-I S_{\Gamma}\right)} \leq 2^{O\left(\log _{2}^{2} m\right)} .
$$

From the above discussions, we know that any separation result between $\chi(G)$ and $\mathbf{b p}(G)$ gives corresponding separation between $N^{0}(C L-I S)$ and the trivial lower bound $\log _{2}|V(\Gamma)|$. We do not know whether the converse is also true yet. However, a weaker converse does exist, as was observed by Alon and Haviv [2]. More precisely, the gap between $N^{0}\left(C L-I S_{\Gamma}\right)$ and $\log _{2}|V(\Gamma)|$ implies a gap between $\chi(H)$ and 2-biclique partition number $\mathbf{b p}_{2}(H)$ for some graph $H$.

Let $\Gamma=(V, E)$ be a graph with vertices $V=\left\{v_{1}, \cdots, v_{m}\right\}$ and consider the following graph $H$. The vertices of $H$ are all the pairs $(C, I)$ such that $C$ is a clique and $I$ is an independent set in $\Gamma$ and $C \cap I=\emptyset$. Two vertices $(C, I)$ and $\left(C^{\prime}, I^{\prime}\right)$ are adjacent if $C \cap I^{\prime} \neq \emptyset$ or $C^{\prime} \cap I \neq \emptyset$. For every vertex $v_{i}$ in $\Gamma$, we define two subsets $U_{i}=\left\{(C, I): v_{i} \in C\right\}$ and $W_{i}=\left\{(C, I): v_{i} \in I\right\}$ of $H$. These subsets have the following properties.

1. $U_{i}$ and $W_{i}$ are disjoint.

2. $\left(U_{i}, W_{i}\right)$ is a complete bipartite subgraph of $H$.

3. $G^{\prime}=\cup_{i=1}^{m} \mathcal{B}\left(U_{i}, W_{i}\right)$ and each edge of $H$ is covered at most two times.

The property (1) holds since $C \cap I=\emptyset$ for any vertex $(C, I)$ of $H$. To verify (2), consider two vertices $(C, I) \in U_{i}$ and $\left(C^{\prime}, I^{\prime}\right) \in W_{i}$. Then $v_{i} \in C \cap I^{\prime}$, which means $C \cap I^{\prime} \neq \emptyset$ and thus $(C, I)$ and $\left(C^{\prime}, I^{\prime}\right)$ are adjacent in $H$. To prove (3), note that by definition, any edge $(C, I) \sim\left(C^{\prime}, I^{\prime}\right)$ in $G^{\prime}$ either satisfies $C \cap I^{\prime} \neq \emptyset$ or $C^{\prime} \cap I \neq \emptyset$ or both. If $C \cap I^{\prime} \neq \emptyset$, then there is a unique $i$ (since $\left.\left|C \cap I^{\prime}\right| \leq 1\right)$ such that $v_{i} \in C$ and $v_{i} \in I^{\prime}$, which means that this edge belongs to $\mathcal{B}\left(U_{i}, W_{i}\right)$. The similar conclusion holds in the case when $C^{\prime} \cap I \neq \emptyset$. Thus every edge of $H$ is covered by $\left\{\mathcal{B}\left(U_{i}, W_{i}\right)\right\}_{i=1}^{m}$ either once or twice. This shows that $\mathbf{b p}_{2}(H) \leq m=|V(\Gamma)|$.

Next we bound the chromatic number of $H$ from below by a function of $N^{0}\left(C L-I S_{\Gamma}\right)$. Denote the matrix of $C L-I S_{\Gamma}$ by $M$. By definition, an independent set $I^{\prime}=\left\{\left(C_{1}, I_{1}\right), \cdots,\left(C_{l}, I_{l}\right)\right\}$ of graph $H$ corresponds to an all-zero submatrix of $M$, whose rows and columns are indexed by $C_{1}, \cdots, C_{l}$ and $I_{1}, \cdots, I_{l}$ respectively. Thus a proper coloring of $H$ corresponds to a covering of the 0-entries of $M$ by monochromatic rectangles. Therefore $\chi(H) \geq C^{0}(M)=C^{0}\left(C L-I S_{\Gamma}\right) \geq 2^{N^{0}\left(C L-I S_{\Gamma}\right)}$ and hence we established the following claim.

Claim 4.2 For every graph $\Gamma$ there exists a graph $H$ such that

$$
\mathbf{b p}_{2}(H) \leq|V(\Gamma)| \quad \text { and } \quad \chi(H) \geq 2^{N^{0}\left(C L-I S_{\Gamma}\right)} .
$$




\section{Concluding remarks}

In this paper we constructed a graph which has a polynomial gap between the chromatic number and the biclique partition number, thereby disproving the Alon-Saks-Seymour conjecture. A very interesting problem which remains widely open is to determine how large this gap can be. In communication complexity it is a long standing open problem to prove an $\Omega\left(\log ^{2} N\right)$ lower bound on the complexity of clique vs. independent set problem for graph on $N$ vertices. Since, as we already explained in the previous section, this problem is closely related to the Alon-Saks-Seymour conjecture, it is plausible to believe that one can obtain a corresponding gap between chromatic and biclique partition numbers. We conjecture that there exists a graph $G$ with biclique partition number $k$ and chromatic number at least $2^{c \log ^{2} k}$, for some constant $c>0$. Existence of such graph will also resolve the complexity of clique vs. independent set problem.

Another intriguing question which deserves further study is to determine the $t$-biclique covering numbers of complete graphs. This will also solve the problem of the maximum possible cardinality of $t$-neighborly family of standard boxes in finite dimensional Euclidean spaces. Even the asymptotics of $\mathbf{b p}_{t}\left(K_{k}\right)$ is only known up to a multiplicative constant factor. In the first open case when $t=2$, the best current bounds are $(1+o(1)) k^{1 / 2} \leq \mathbf{b p}_{2}\left(K_{k}\right) \leq(1+o(1)) 2 k^{1 / 2}$ and it would be interesting to close this gap.

Acknowledgment. We would like to thank Noga Alon for explaining to us his results with I. Haviv on connection between the Alon-Saks-Seymour conjecture and the clique vs. independent set problem.

\section{References}

[1] N. Alon, Neighborly families of boxes and bipartite coverings, Algorithms and Combinatorics 14 (1997), 27-31.

[2] N. Alon and I. Haviv, private communication.

[3] N. Alon and P. Seymour, A counterexample to the rank-coloring conjecture, Journal of Graph Theory 13 (1989), 523-525.

[4] L. Babai and P. Frankl, Linear algebra methods in combinatorics with applications to geometry and computer science, The University of Chicago, 1992.

[5] S. Fajtlowicz. On conjectures of Graffiti II, Congressus Numeratum 60 (1987), 189-198.

[6] Z. Gao, McKay B.D., R. Naserasr and B. Stevens, On Alon-Saks-Seymour Conjecture, to appear.

[7] R. L. Graham and H. O. Pollak, On embedding graphs in squashed cubes, in: Graph theory and applications, Lecture Notes in Math. 303, Springer, Berlin, 1972, 99-110.

[8] J. Kahn, Coloring nearly-disjoint hypergraphs with $n+o(n)$ colors, J. Combin. Theory Ser. A 59 (1992), 31-39. 
[9] J. Kahn, Recent results on some not-so-recent hypergraph matching and covering problems, in: Extremal problems for finite sets (Visegrád, 1991), Bolyai Soc. Math. Stud. vol. 3, János Bolyai Math. Soc., Budapest, 1994, 305-353.

[10] E. Kushilevitz, N. Linial and R. Ostrovsky, The linear-array conjecture in communication complexity is false, Combinatorica 19 (1999), 241-254.

[11] E. Kushilevitz and N. Nisan, Communication complexity, Cambridge University Press, Cambridge, 1997.

[12] L. Lovász and M. Saks, Lattices, Mobius functions, and communication complexity, Journal of Computer and System Sciences 47 (1993), 322-349.

[13] D. Mubayi and S. Vishwanathan, Biclique Coverings and the Chromatic Number. The Electronic Journal of Combinatorics 16(1) (2009), N34.

[14] N. Nisan and A. Wigderson, On rank vs. communication complexity, Combinatorica 15 (1995), $557-565$.

[15] G. Peck, A new proof of a theorem of Graham and Pollak, Discrete Math. 49 (1984), 327-328.

[16] R. Raz and B. Spieker, On the "log rank"-conjecture in communication complexity, Combinatorica 15 (1995), 567-588.

[17] A. Razborov, The gap between the chromatic number of a graph and the rank of its adjacency matrix is superlinear, Discrete Math. 108 (1992), 393-396.

[18] H. Tverberg, On the decomposition of $K_{n}$ into complete bipartite graphs, J. Graph Theory 6 (1982), 493-494.

[19] C. Van Nuffelen, Rank, Clique, and Chromatic Number of a Graph, System Modeling and Optimization, 38, Lect. Notes Control Inf. Sci, 605-611.

[20] S. Vishwanathan, A polynomial space proof of the Graham-Pollak theorem, J. Combin. Theory Ser. A 115 (2008), 674-676.

[21] M. Yannakakis, Expressing combinatorial optimization problems by linear programs, Journal of Computer and System Sciences 43 (1991), 441-466.

[22] A. Yao, Some complexity questions related to distributive computing, Proceedings of the $11^{\text {th }}$ ACM STOC, ACM New York (1979), 209-213.

[23] J. Zaks, Bounds of neighborly families of convex polytopes, Geometriae Dedicata 8 (1979), 279-296. 